\title{
Densidad mineral ósea en escolares nacidos prematuros
}

\author{
Aída Milinarsky T, Sylvia Fischer G, Vinka G iadrosich R, \\ María Isabel Hernández C, María Teresa Torres C.

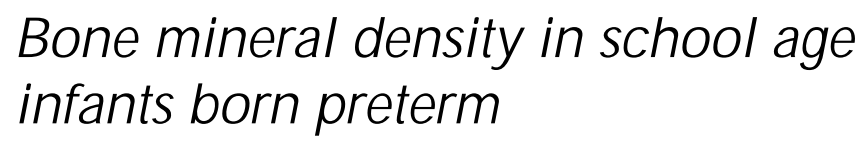

Background: The age at which children born preterm normalize their bone mineral density, is not well known. Aim: To study if children born preterm have normalized their bone mineral density at age 5 to 7 years. Patients and methods: Twenty six infants born preterm (14 male), were studied at age 5 to 7 years. Birth weight, present weight and height, bone age, calcium and phosphate intake at the first year of life and at the current age were assessed. Bone mineral density was measured by single photon $\mathrm{X}$ ray absorptiometry in the dominant forearm. A blood sample was obtained to measure insulin growth factor 1 (IGF-1). As a control group, 105 healthy age-paired infants born at term, were studied. Results: Bone mineral density was significantly lower in infants born preterm than in their term counterparts $\left(0.273 \pm 0.01 \mathrm{~g} / \mathrm{cm}^{2}\right.$ and $0.302 \pm 0.01 \mathrm{~g} / \mathrm{cm}^{2}$ respectively, $\left.\mathrm{p}<0.001\right)$. There was a positive correlation between bone mineral density and IGF-1 $(r=0.49, \mathrm{p}=0.01)$. No correlation with the other measured parameters was observed. Conclusions: Infants that were born preterm have a lower bone mineral density at 5 to 7 years of age than their term controls. Bone mineral density correlates with IGF-1 (Rev Méd Chile 2003; 131: 1289-94).

(Key Words: Birth weight; Bone density; Bone development; Delivery)

Recibido el 24 de enero, 2003. Aceptado en versión corregida el 1 de septiembre, 2003. Departamentos de Pediatría y Medicina, Facultad de Medicina, Universidad de Valparaíso. Instituto Médico Infantil, Hospital Carlos van Buren, Hospital Gustavo Fricke.

C on los avances en los cuidados intensivos neonatales, la tasa de sobrevida de los niños nacidos prematuros ha aumentado y los pediatras deben estar alertas a las eventuales patologías que podnían surgir posteriormente en la vida de estos niños. En Chile la sobrevida de los niños nacidos prematuros de 26 semanas de gestación ya alcanza a 50\% ${ }^{1}$.

La acreción de nutrientes como calcio, fósforo y otros minerales ocurre predominantemente en el tercer trimestre de la vida intrauterina ${ }^{2}$, por lo tanto, los niños nacidos prematuros tienen alto riesgo de desarrollar déficit de mineralización

Correspondencia a: Dra. Aída Milinarsky. Departamento de Pediatría, Universidad de Valparaíso. Calle Hontaneda 2653, Oficina 406 - Casilla 92-V, Valparaíso, Chile. Fax: 32507360. E mail: pediatria@uv.cl ósea ${ }^{2,3}$. La ganancia de masa mineral ósea durante la infancia es un proceso complejo que involucra determinantes genéticos, hormonales, factores nutricionales y ambientales ${ }^{4-6}$. Son variables relevantes en los niños nacidos prematuros la edad gestacional, el peso de nacimiento, los aportes nutricionales ${ }^{7,8}$, la actividad física ${ }^{9}$, la intercurrencia de algunas enfermedades, administración de determinados medicamentos y factores hormonales ${ }^{10}$. La edad a la cual el crecimiento compensatorio o recuperacional (catch-up) de la densidad mineral ósea (DMO) se completa no está establecida11-13.

La literatura muestra normalización de DMO a diferentes edades: a las 40 semanas post concepcionales ${ }^{11}$, desde los 2 años ${ }^{5}$; desde los 3-4 años ${ }^{4}$ y de los 8 años ${ }^{12,13}$. Entre los determinantes hormonales, 
la somatomedina C plasmática (IGF-I) ha sido foco de extensa investigación debido a su participación como promotor de crecimiento y diferenciación celular y también por ser un determinante mayor en la aposición de la matriz ósea. Algunos autores han sugerido una relación entre niveles elevados de IGFI y ganancia ósea durante la infancia ${ }^{10}$.

El objetivo de este estudio fue determinar si los niños de 5 a 7 años nacidos prematuros han normalizado su masa mineral ósea y correlacionarla con la edad gestacional, peso de nacimiento, ingestión de calcio y fósforo durante el primer año de vida, actual ingesta de calcio, edad ósea y niveles plasmáticos de IGF-I.

\section{PACIENTES Y MÉTODO}

Pacientes: La población estudiada incluyó escolares nacidos prematuros (ENP) con peso adecuado para su edad gestacional, de 34 o menos semanas de gestación, nacidos entre julio de 1992 y diciembre de 1995, en los hospitales Carlos van Buren de Valparaíso, Gustavo Fricke de Viña del Mar y Clínica Reñaca, centros de referencia para atención de recién nacidos prematuros de las ciudades de Viña del Mar y Valparaíso. De la revisión de las fichas clínicas se obtuvieron los datos del período perinatal. Se excluyeron los niños con enfermedades congénitas, infecciones, enfermedades metabólicas, respiratorias, digestivas y óseas, por lo tanto se incluyeron sólo los niños que requirieron asistencia médica mínima. Fueron citados por correo 102 niños que cumplían con los criterios de inclusión. De ellos 26 aceptaron participar en el estudio.

El grupo control lo constituyeron 105 niños sanos prepuberales nacidos de término, con peso adecuado para la gestación que participaron en un estudio previo ${ }^{14}$.

Método: Los datos de semanas de gestación al momento de nacer, peso de nacimiento, asistencia médica y nutricional del período neonatal, se obtuvieron de las fichas clínicas. Los padres contestaron un cuestionario que incluyó: ingestión de calcio, fósforo y vitamina D durante el primer año de vida; consumo de calcio actual, que se calculó por el método recordatorio de la ingestión alimentaria de los últimos 3 días; actividad física, que se consideró normal si el niño cumplía con las clases de educación física, disminuida si estaban eximidos y aumentada si además tenían actividad extracurricular. Se les hizo examen físico. Se midió la estatura en $\mathrm{cm}$ y el peso en $\mathrm{kg}$ en balanza marca Secca ${ }^{\circledR}$, descalzos y con ropa liviana. Se consideró el promedio de tres mediciones consecutivas efectuadas por el mismo operador. Se calculó el índice de masa corporal (IMC) según la fórmula peso en $\mathrm{kg}$ dividido por el cuadrado de la estatura en metros. Los resultados fueron expresados en puntaje $z$ en base al grupo control.

Variables óseas: la edad ósea fue evaluada con radiografía de mano izquierda de acuerdo a atlas de Greulich y Pyle ${ }^{15}$.

La DMO $\left(\mathrm{g} / \mathrm{cm}^{2}\right)$ fue medida por absorciometría de rayos X de 1 fotón (Osteometer DTX-100) a nivel radio cubital del brazo dominante. El examen lo realizó un solo operador y el cuociente de variación para 5 medidas repetidas del fantoma que provee el fabricante fue $0,7 \%$ in vitro. El cuociente de variación para 3 medidas repetidas en el mismo sujeto, el mismo día, realizado en 5 niños de diferentes edades fue $3 \%$ in vivo. Los resultados fueron comparados con el grupo control $^{14} \mathrm{y}$ expresados en puntaje $\mathrm{z}$.

Determinaciones plasmáticas de IGF-I. Se midió en plasma venoso por ensayo radioinmunométrico (IRMA). El procedimiento emplea el principio radioinmunométrico de 2 sitios. La técnica (DSL-5600 IGF-I IRMA) incluye una extracción simple en la cual se separa IGF-I de su proteína ligante en el suero. El IRMA es un ensayo no competitivo de 2 anticuerpos. El primer anticuerpo es inmovilizado en la pared interna de los tubos. El otro anticuerpo es radiomarcado para detección. La hormona presente en la muestra del paciente, en la muestra estandarizada y en la muestra control es ligada por los 2 anticuerpos para formar un complejo "sandwich". Los materiales no ligados son removidos por decantación y por lavado de los tubos. La sensibilidad teórica es 0,80 ng/ml. Precisión intra ensayo: se determinó del promedio $(\overline{\mathrm{x}})$ de 8 duplicados.

$\begin{array}{lcccc}\text { Muestra } & \mathrm{n} & \overline{\mathrm{x}} \mathrm{ng} / \mathrm{ml} & \mathrm{DS} \mathrm{ng} / \mathrm{ml} & \begin{array}{c}\text { coeficiente } \\ \text { de variación \% }\end{array} \\ \text { I } & 8 & 9,4 & 0,3 & 3,4 \\ \text { II } & 8 & 55,4 & 1,6 & 3 \\ \text { III } & 8 & 263,6 & 3,9 & 1,5\end{array}$


Precisión interensayo: se determinó del promedio de 8 duplicados.

$\begin{array}{lcccc}\text { Muestra } & \mathrm{n} & \overline{\mathrm{x}} \mathrm{ng} / \mathrm{ml} & \mathrm{DS} \mathrm{ng} / \mathrm{ml} & \begin{array}{c}\text { coeficiente } \\ \text { de variación \% }\end{array} \\ \text { I } & 8 & 10,4 & 0,9 & 8,2 \\ \text { II } & 8 & 53,8 & 0,8 & 1,5 \\ \text { III } & 8 & 255,9 & 9,6 & 3,7\end{array}$

Especificidad: La reacción cruzada de IGF-I en suero ha sido medida contra los siguientes péptidos humanos: IGF-I 100\%; IGF-II, insulina, pro insulina y hormona del crecimiento no detectable.

Los valores fueron comparados con los valores de referencia para población infantil proporcionados por el laboratorio.

Análisis estadístico: incluyó prueba para datos normales Kolmogorov-Smirnov, prueba t de Student, prueba de correlación (Pearson y Spearman), prueba de independencia (chi cuadrado), ANOVA y análisis de regresión lineal. El error $\alpha$ se estableció en 0,05 para todas las pruebas. Este estudio transversal descriptivo fue aprobado por el Comité de Ética de la Escuela de Medicina de la Universidad de Valparaíso.

\section{RESUlTADOs}

De los 102 ENP citados por correo completaron el estudio 26 (14 niños y 12 niñas), de entre 5 y 7 años 9 meses de edad. La edad gestacional al nacer fluctuó entre 25 y 34 semanas y el peso de nacimiento entre 740 y $2.200 \mathrm{~g}$ (Tabla 1).

La ingesta promedio de calcio en el primer año de vida de los ENP fue $511 \pm 60 \mathrm{mg} /$ día y la de fósforo fue $377 \pm 57 \mathrm{mg} /$ día. Diecinueve niños habían ingerido suplemento de vitamina D en el primer año de vida. La ingesta promedio actual de calcio era $730 \pm 194 \mathrm{mg} /$ día para los ENP versus $618 \pm 232 \mathrm{mg} /$ día del grupo control $(\mathrm{p}=<0,025)$.

Todos los niños tenían actividad física normal. Las edades óseas estaban en rangos de normalidad.

La estatura de los niños resultó normal (Tabla 1). El promedio de los z score de estatura fue $-0,004 \pm 1,0, p=0,5$.

El promedio de los z score de IMC para los ENP de ambos sexos fue $0,39 \pm 1,2, p=0,051$. En los varones $0,16 \pm 1,2, p=0,3$, similar al grupo control. Las niñas nacidas prematuras tenían $\mathrm{z}$ score de IMC significativamente mayor que las niñas nacidas de término, $z=0,67 \pm 1,2, p=0,04$.

Los ENP tenían DMO significativamente menor $(\overline{\mathrm{x}}$ $0,273 \mathrm{~g} / \mathrm{cm}^{2} \pm 0,01$ ) comparada con las DMO del grupo control $\left(\bar{x} 0,302 \mathrm{~g} / \mathrm{cm}^{2} \pm 0,01\right), p=0,0003$. El promedio de los z score de las DMO de los ENP fue $0,72 \pm 0,93, p=0,000275$. Esta diferencia se mantuvo al considerar el sexo: los niños varones nacidos prematuros $\left(\overline{\mathrm{x}} 0,284 \mathrm{~g} / \mathrm{cm}^{2} \pm 0,04\right)$ comparado con varones nacidos de término $\left(\overline{\mathrm{x}} 0,320 \mathrm{~g} / \mathrm{cm}^{2} \pm 0,04\right), \mathrm{p}=0,0014$; el promedio de las DMO de las niñas nacidas prematuras $\left(\overline{\mathrm{x}} 0,261 \mathrm{~g} / \mathrm{cm}^{2} \pm 0,02\right)$ fue menor comparado con las niñas nacidas de término $\left(\overline{\mathrm{x}} 0,285 \mathrm{~g} / \mathrm{cm}^{2}\right.$ $\pm 0,04) \mathrm{p}=0,04$ (Tabla 1). El promedio de los z score de las DMO de los ENP en varones fue $-0,84 \pm 0,85$, $\mathrm{p}=0,001$; en las mujeres fue $-0,58 \pm 1,04, p=0,04$.

\section{Tabla 1. C aracterísticas demográficas y antropométricas y densitometría ósea en escolares nacidos prematuros y controles}

\begin{tabular}{|c|c|c|c|c|c|c|c|c|}
\hline \multirow{3}{*}{ Variables } & \multicolumn{4}{|c|}{ Niños } & \multicolumn{4}{|c|}{ Niñas } \\
\hline & \multicolumn{2}{|c|}{$\begin{array}{l}\text { Pretérmino } \\
\quad(\mathrm{n}=14)\end{array}$} & \multicolumn{2}{|c|}{$\begin{array}{l}\text { Control } \\
(\mathrm{n}=56)\end{array}$} & \multicolumn{2}{|c|}{$\begin{array}{l}\text { Pretérmino } \\
\quad(\mathrm{n}=12)\end{array}$} & \multicolumn{2}{|l|}{$\begin{array}{c}\text { Control } \\
(n=49)\end{array}$} \\
\hline & Promedio & D.E. & Promedio & D.E. & Promedio & D.E. & Promedio & D.E \\
\hline Edad gestacional (semanas) & $32 *$ & 1,5 & & & $30,7 *$ & 3,1 & & \\
\hline Peso nacimiento $(\mathrm{g})$ & 1787* & 301 & 3350 & 362 & $1618^{*}$ & 390 & 3248 & 384 \\
\hline Edad actual (años) & 6,3 & 0,7 & 6,0 & 0,8 & 6,4 & 0,7 & 6,2 & 0,8 \\
\hline Peso actual (kg) & 22,4 & 5,8 & 24,4 & 4,8 & 23,2 & 4,9 & 24,8 & 4,4 \\
\hline Estatura actual $(\mathrm{cm})$ & 117,6 & 8,6 & 119,8 & 7,6 & 117,3 & 6,6 & 119,9 & 6,9 \\
\hline Ingesta de calcio (g/día) & $739 *$ & 182 & 662 & 190 & $718^{*}$ & 216 & 567 & 222 \\
\hline DMO $\left(\mathrm{g} / \mathrm{cm}^{2}\right)$ & $0,284 *$ & 0,04 & 0,320 & 0,04 & $0,261 *$ & 0,02 & 0,285 & 0,04 \\
\hline
\end{tabular}

$\mathrm{DE}=$ desviación estándar. $\quad *=\mathrm{p}<0,05$ comparado con control. 
Los niveles plasmáticos de IGF-I de los ENP estuvieron en rangos de normalidad, pero el valor promedio fue significativamente mayor $(\bar{x} 228,7$ $\mathrm{ng} / \mathrm{ml} \pm 80,4$ ) comparados con los controles nacidos de término ( $\overline{\mathrm{x}} 169,8 \mathrm{ng} / \mathrm{ml} \pm 119,9) \mathrm{p}=0,048$.

En los ENP, los niveles de IGF-I plasmáticos se correlacionaron en forma positiva y significativa con el peso de nacimiento: $r=0,60 p=0,001$; con el IMC: $r=0,60 p=0,01$; con la edad ósea $r=0,53$ $p=0,005$; y con la estatura: $r=0,41, p=0,04$.

Los valores z de DMO de los ENP tuvieron correlación positiva sólo con los niveles plasmáticos de IGF-I: $r=0,44 p=0,01$ (Figura 1). No se encontró correlación con las siguientes variables: edad gestacional, peso de nacimiento, ingesta de calcio y fósforo en el primer año de vida; ingesta de calcio ni edad ósea al momento del estudio.

\section{Discusión}

En este estudio los ENP a la edad de 5 a 7 años tienen menor DMO que los niños nacidos de término de igual edad, habiendo alcanzado la normalidad de talla y peso. Hay controversia respecto a la edad en que normalizan la densidad mineral ósea los niños nacidos prematuros. Zamo$\mathrm{ra}^{13}$, en un estudio en niñas escolares nacidas prematuras de 7-9 años describe normalización de la estatura, peso y DMO en huesos predominantemente corticales, pero menor mineralización en cuello femoral, metafisis radial y cadera. Ryan ${ }^{2}$, en
46 niños de 8 años de edad nacidos prematuros, usando absorciometría ósea de fuente radiológica de doble haz (DEXA) en columna lumbar, antebrazo y cuello femoral, describe menor contenido y densidad mineral ósea en todos estos sitios. A diferencia de nuestros ENP que habían normalizado talla y peso, los niños nacidos prematuros del grupo de Ryan tenían menor peso (2,5 kg menos) y menor estatura (3,7 cm menos), comparado con el grupo control. Congdon ${ }^{11}$, con absorciometría ósea de un fotón, encontró normalización total de la masa ósea del antebrazo ya a las 25-50 semanas después de la fecha en que habrían completado su gestación de término, aunque la mayonía la normalizaron en las primeras 12 semanas después de la fecha de gestación de término. Estos hallazgos fueron confirmados por DEXA a nivel de la columna lumbar en los primeros años de vida4. Ichiba $^{5}$ en estudio prospectivo en niños de muy bajo peso para la edad gestacional demostró que a los 2 años de edad había normalización de la DMO a nivel lumbar. Fewtrell ${ }^{16}$ en 244 niños nacidos prematuros evaluó la DMO en 3 etapas del desarrollo: a los 18 meses, a los $71 / 2$ a 8 años y entre los 8 y 12 años, demostrando asociación entre crecimiento y ganancia de masa ósea, concluyendo que aquel niño que sube de canal de crecimiento es el que logra mayor masa ósea. Hasta la edad de 8 años, también observó una menor DMO en los ENP que resultaron de talla y peso menor a lo normal. Es razonable que haya aplicado un factor de corrección por el tamaño corporal, ya que

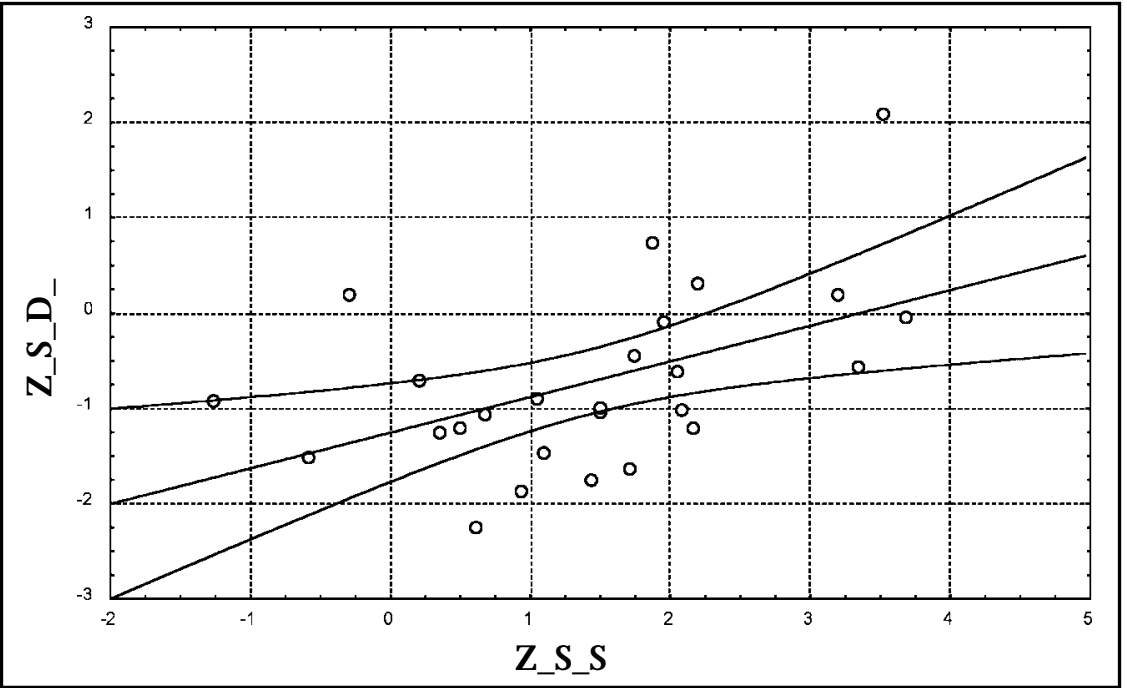

Figura 1. Correlación entre DMO de niños nacidos prematuros. Niveles plasmáticos de IGF-I.

$\mathrm{r}=0,4986$

$\mathrm{p}=0,01$

$\mathrm{z} \mathrm{s} \mathrm{d}=\mathrm{z}$ score de DMO $\mathrm{z}$ s s= z score de IGF-I 
además los niños puberales tienen crecimiento variable, influido por su estadio puberal. Nuestro grupo de ENP tiene entre 5 y 7 años, sin diferencia significativa de talla con sus pares nacidos de término por lo que no se efectuó correcciones.

El estudio fue diseñado para abarcar la mayor cantidad de pacientes del universo seleccionado, recurriendo a la citación por correo al domicilio registrado en la ficha clínica. Si bien sólo 26 niños se incorporaron al estudio, las causas de la no participación son fortuitas como cambio de domicilio, falta de interés de los padres, falta de tiempo disponible para concurrir al control y otros que consideramos no afectarían la representatividad de la muestra.

Se empleó la absorciometría ósea periférica radiológica por ser un método preciso, rápido, de bajo costo y muy baja irradiación, lo que lo hace apropiado para su empleo en niños ${ }^{17}$. Los errores de precisión son similares a los equipos de doble fotón y medición central ${ }^{18}$ que son los más ampliamente usados en nuestro país. Mide predominantemente hueso cortical que corresponde al $85 \%$ del esqueleto. Si bien es poco sensible a los cambios metabólicos es un buen método para estudio poblacional para seleccionar individuos en riesgo ${ }^{19}$. Se comparó con valores normales del mismo equipo ${ }^{14}$.

Referente a la nutrición, Hori ${ }^{4}$ describió que la ingestión mineral en 21 niños nacidos prematuros permanecía baja en los primeros años de vida y que se mantenían osteopénicos después de la fecha en que habrían completado su gestación, sin embargo, a la edad de 3-4 años todos los niños habían normalizado su densidad y contenido mineral óseo, sugiriendo una resolución espontánea de la osteopenia lumbar y que esto ocurriría en los primeros años de vida.

En nuestros ENP la baja DMO no se correlacionó con la ingestión de calcio, fósforo y vitamina $\mathrm{D}$ en el primer año de vida ni con su ingestión actual. Nuestros pacientes habían recibido suficiente calcio para cumplir con las recomendaciones chilenas e internacionales ${ }^{20-22}$. Esto se contrapone con lo que anteriormente habíamos descrito en escolares chilenos normales nacidos de término en quienes encontramos una ingesta deficitaria de calcio $^{14}$. Creemos que estos niños pueden estar afectos a una vigilancia nutricional más estricta por los padres y por el equipo médico que explique su adecuada ingesta de calcio en edad escolar.

Aunque se ha descrito menor crecimiento longitudinal en niños nacidos prematuros ${ }^{23,24}$, nuestros ENP, al igual que lo referido por otros autores $^{25,26}$, habían alcanzado estatura normal.

En nuestro trabajo, las niñas nacidas prematuras tuvieron significativo mayor IMC que las congéneres nacidas de término. Este hallazgo concuerda con lo descrito en la última década en la población infantil chilena ${ }^{27}$ : hay aumento del IMC, especialmente en las niñas, hecho que constituye una preocupación para las autoridades nacionales de salud.

Intentando explicar cuál pudiera ser la o las señales biológicas para la aceleración del crecimiento de la masa mineral ósea en los niños nacidos prematuros medimos IGF-I plasmática. La IGF-I actúa como una hormona trófica sobre el hueso, afectando en forma positiva su crecimiento y recambio, estimulando la síntesis de colágeno por los osteoblastos y el crecimiento óseo longitudinal $^{10}$. La IGF-I ha demostrado comportarse como un marcador nutricional ya que presenta correlación positiva con la estatura, el peso y la edad ósea. Se han descrito diferentes métodos para determinar IGF-I en plasma, lo cual dificulta la interpretación y comparación de los diferentes estudios publicados, por lo cual se hace necesario que la Organización Mundial de la Salud diseñe un preparado basado en IGF-I recombinante humana ${ }^{28}$.

Nuestros pacientes nacidos prematuros que tenían mayor DMO tenían claramente niveles más altos de IGF-I.

En conclusión, los niños nacidos prematuros estudiados con edades entre 5 y 7 años 9 meses tienen estatura, IMC y edad ósea similares a los niños nacidos de término. Los ENP tiene DMO menor a lo normal sin que esta situación se correlacione con la ingesta alimentaria de minerales en el primer año de vida, con el peso de nacimiento, la edad ósea, ni la ingesta de calcio en el momento del estudio. Los niveles plasmáticos de IGF-I fueron más altos en los niños nacidos prematuros y mostraron una correlación positiva con la DMO. 


\section{REFERENCIAS}

1. HÜBNER ME, RAMíREZ R. Sobrevida, viabilidad y pronóstico del prematuro. Rev Méd Chile 2002; 130: 931-8.

2. RYAN S. Bone Mineralization in Preterm Infants. Nutrition 1998; 14: 745-7.

3. Horsman A, Ryan SW, Congdon PJ, Truscott JG, JAMES JR. Osteopenia in extremely low-birthweight infants. Arch Dis Child 1989; 64: 485-8.

4. Hori $\mathrm{C}$, Tsukahara H, Fuji $Y$, Kawamitsu T, Konishi $Y$, Yамамото $\mathrm{K}$ ET AL. Bone mineral status in pretermborn-children assessment by dual energy X-ray absorptiometry. Biol Neonate 1995; 68: 254-8.

5. Ichiba H, Shintaku H, Fujimaru M, Hirai C, Okano Y, Funato M. Bone mineral density of the lumbar spine in very-low-birth-weight infants: longitudinal study. Eur J Pediatr 2000; 159: 215-8.

6. Sale BL, Glorieux FH. Assessment of bone mineral content in infants. The new age. Acta Paediatr 1993; 82: 709-10.

7. Bishop NJ. Early diet of preterm infants and bone mineralization at age five years. Acta Paediatr 1996; 85: 230-6.

8. MoriEy R. Randomized diet in the neonatal period and growth performance until 7.5-8 years of age in preterm children. Am J Clin Nutr 2000; 71: 822-8.

9. Moyer-Mileur L Effect of physical activity on bone mineralization in premature infants. J Pediatr 1995; 127: 620-5.

10. SOYKa LA, FAIRFIEID W, KuBANSKI A. Hormonal Determinants and Disorders of Peak Bone Mass in Children. J Clin Endocrinol Metab 2000; 85: 3951-63.

11. Congdon PJ, Horsman A, Ryan SW, Truscott JG, DURWARD H. Spontaneous resolution of bone mineral depletion in preterm infants. Arch Dis Child 1990; 65: 1038-42.

12. Bowden LS, Jones CJ, Ryan SW. Bone mineralization in preterm infant aged 8 years. Eur J Pediatr 1999; 158: 658-61.

13. Zamora SA, Be山i DC, Rzzzol R, Slosman DO, Bonjour JP. Lower Femoral Neck Bone Mineral Density in Prepubertal Former Preterm Girls. Bone 2001; 29: 424-7.

14. Milinarsky A, Fischer S, Giadrosich V, Casanova D. Bone mineral density by single X-Ray aborptiometry in Chilean children and adolescent. J Rheumatol 1998; 25: 2003-8.

15. GREULCH WW, Pyle SI. Radiographic atlas of skeletal development of the hand and wrist. 2nd ed. Stanford CA: Stanford University Press; 1959.

16. Fewtrell MS, Prentice A, Cole TJ, Lucas A. Effects of growth during infancy and childhood on bone mineralization and turnover in preterm children aged 8-12 years. Acta Paediatr 2000; 89: 148-53.

17. Genant H, Engelke K, Fuerst T, GlüEr C, Gramp $\mathrm{S}$, HaRRIS S ET aL. Noninvasive Assessment of Bone Mineral and Structure: State of Art. Journal J Bone Miner Res 1996; 11: 707-30.

18. MUIER P. Bone mineral density-clinical use and application. J Endocrinol Metab Clin NAm 2003; 32: 159-79.

19. Damilakis J, Papadokostakis G, Perisinakis K, Hadjipavlou A, Gourtsoyiannis N. Can radial bone mineral density and quantitative ultrasound measurements reduce the number of women who need axial density skeletal assessment? Osteoporos Int $2003 \mathrm{Jul} 24$ [Epub ahead of print].

20. Ministerio de Salud. Programa Infantil y del Adolescente; UNICEF. Santiago de Chile: MINSAL, 1993.

21. NIH Consensus Development Panel on Optimal Calcium Intake. JAMA 1994; 272: 1942-8.

22. American Academy of Pediatrics. Calcium requirements of infants, children and adolescents. Pediatrics 1999; 104: 1152-7.

23. Peralta-Carcelen M, Jackson DA, Goran M, Royal S, Mayo M, Nelson K. Growth of adolescents who were born at extremely low birth weight without major disability. J Pediatr 2000; 136: 633-40.

24. Hack M, Merkatz IR, McGrath SK, Jones PK, FANAROFF AA. Catch-up growth in very low-birthweight infants. AJDC 1984; 138: 370-5.

25. Kitchen WH, Doyle LW, Ford GW, Callanan C. Very low birth weight and growth to age 8 years I: weight and height. AJDC 1992; 146: 40-5.

26. StJeRnQvist K, SuENNINGSEN NW. Extremely lowbirth-weight infants less than $901 \mathrm{~g}$. Growth and development after one year of life. Acta Paediatr 1993; 82: 40-4.

27. Muzzo S, Cordero J, Burrows R. Changes in the prevalence of overweight in school age Chilean children during the last 8 years. Rev Chil Nutr 1999; 26: 311-5.

28. Mora S, Pitukcheewanont P, Neison JC, Gilsanz V. Serum Levels of Insulin-Like Growth Factor I and the Density, Volume, and Cross-Sectional Area of Cortical Bone in Children. J Clin Endocrinol Metab 1999; 84: 2780-3.

\section{Agradecimientos}

Los autores agradecen a Raquel Ravest por su cooperación; a María Paz Casanova por su asistencia estadística; a Gabriela González por su ayuda como secretaria y a Renate Hulse por su ayuda a incorporar niños al protocolo. 